\title{
Cannabidiol for psychosis: A review of 4 studies
}

Sy Atezaz Saeed, MD, MS, and Kathryn E. Clary, BA

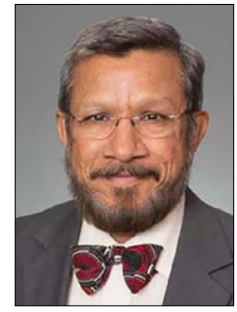

Sy Atezaz Saeed, MD, MS

Department Editor

Some early research

suggests CBD

might be beneficial

for patients with

psychosis, but

evidence is mixed

\section{$f$}

Discuss this article at www.facebook.com/ MDedgePsychiatry

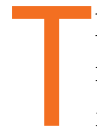
here has been increasing interest in the medicinal use of cannabidiol (CBD) for a wide variety of health conditions. CBD is one of more than 80 chemicals identified in the Cannabis sativa plant, otherwise known as marijuana or hemp. Delta-9-tetrahydrocannabinol (THC) is the psychoactive ingredient found in marijuana that produces a "high." CBD, which is one of the most abundant cannabinoids in Cannabis sativa, does not produce any psychotomimetic effects.

The strongest scientific evidence supporting CBD for medicinal purposes is for its effectiveness in treating certain childhood epilepsy syndromes that typically do not respond to antiseizure medications. Currently, the only FDA-approved CBD product is a prescription oil cannabidiol (brand name: Epidiolex) for treating 2 types of epilepsy. Aside from Epidiolex, state laws governing the use of $\mathrm{CBD}$ vary. $\mathrm{CBD}$ is being studied as a treatment for a wide range of psychiatric conditions, including bipolar disorder, schizophrenia, dystonia, insomnia, and anxiety. Research supporting CBD's benefits is limited, and the US National Library of Medicine's MedlinePlus indicates there is "insufficient evidence to rate effectiveness" for these indications. ${ }^{1}$

Despite having been legalized for medicinal use in many states, CBD is classified as a Schedule I controlled substance by the US Drug Enforcement Agency. Because of this classification, little has been done to regulate and oversee the sale of products containing CBD. In a 2017 study of 84 CBD products sold by 31 companies online, Bonn-Miller et $\mathrm{al}^{2}$ found that nearly $70 \%$ percent of products were inaccurately labeled. In this study, blind testing found that only approximately $31 \%$ of products contained within $10 \%$ of the amount of CBD that was listed on the label. These researchers also found that some products contained components not listed on the label, including THC. ${ }^{2}$

The relationship between cannabis and psychosis or psychotic symptoms has been investigated for decades. Some recent studies that examined the effects of CBD on psychosis found that individuals who use CBD may experience fewer positive psychotic symptoms compared with placebo. This raises the question of whether CBD may have a role in the treatment of schizophrenia and other psychotic disorders. One of the first studies on this issue was conducted by Leweke et $\mathrm{al}_{1}^{3}$ who compared oral CBD, up to $800 \mathrm{mg} / \mathrm{d}$, with the antipsychotic amisulpride, up to $800 \mathrm{mg} / \mathrm{d}$, in 39 patients with an acute exacerbation of psychotic symptoms. Amisulpride is used outside the United States to treat psychosis, but is FDAapproved only as an antiemetic. Patients were treated for 4 weeks. By Day 28, there was a significant reduction in positive symptoms as measured using the Positive and Negative Syndrome Scale (PANSS), with

Dr. Saeed is Professor and Chair, Department of Psychiatry and Behavioral Medicine, East Carolina University Brody School of Medicine, Greenville, North Carolina. Ms. Clary is a student, MD candidate class of 2021, East Carolina University Brody School of Medicine, Greenville, North Carolina.

Disclosures

The authors report no financial relationships with any companies whose products are mentioned in this article, or with manufacturers of competing products. 
no significant difference in efficacy between the treatments. Similar findings emerged for negative, total, and general symptoms, with significant reductions by Day 28 in both treatment arms, and no significant betweentreatment differences.

These findings were the first robust indication that CBD may have antipsychotic efficacy. However, of greater interest may be CBD's markedly superior adverse effect profile. Predictably, amisulpride significantly increased extrapyramidal symptoms (EPS), weight gain, and prolactin levels from baseline to Day 28. However, no significant change was found in any of these adverse effects in the CBD group, and the between-treatment difference was significant (all $P<.01$ ).

Here we review 4 recent studies that evaluated CBD as a treatment for schizophrenia. These studies are summarized in the Table $^{4-7}$ (page 26).

1. McGuire $P$, Robson $P$, Cubala WJ, et al. Cannabidiol (CBD) as an adjunctive therapy in schizophrenia: a multicenter randomized controlled trial. Am J Psychiatry. 2018;175(3):225-231.

Antipsychotic medications act through blockade of central dopamine D2 receptors. For most patients, antipsychotics effectively treat positive psychotic symptoms, which are driven by elevated dopamine function. However, these medications have minimal effects on negative symptoms and cognitive impairment, features of schizophrenia that are not driven by elevated dopamine. Compounds exhibiting a mechanism of action unlike that of current antipsychotics may improve the treatment and outcomes of patients with schizophrenia. The mechanism of action of CBD is unclear, but it does not appear to involve the direct antagonism of dopamine receptors. Human and animal research study findings indicate that CBD has antipsychotic properties. McGuire et $\mathrm{al}^{4}$ assessed the safety and effectiveness of CBD as an adjunctive treatment of schizophrenia.

\section{Study design}

- In this double-blind parallel-group trial conducted at 15 hospitals in the United Kingdom, Romania, and Poland, 88 patients with schizophrenia received CBD $(1,000$ $\mathrm{mg} / \mathrm{d} ; \mathrm{N}=43)$ or placebo $(\mathrm{N}=45)$ as adjunct to the antipsychotic medication they had been prescribed. Patients had previously demonstrated at least a partial response to antipsychotic treatment, and were taking stable doses of an antipsychotic for $\geq 4$ weeks.

- Evaluations of symptoms, general functioning, cognitive performance, and EPS were completed at baseline and on Days 8, 22, and 43 ( \pm 3 days). Current substance use was assessed using a semi-structured interview, and reassessed at the end of treatment.

- The key endpoints were the patients' level of functioning, severity of symptoms, and cognitive performance. Participants were assessed before and after treatment using the PANSS, the Brief Assessment of Cognition in Schizophrenia (BACS), the Global Assessment of Functioning scale (GAF), and the improvement and severity scales of the Clinical Global Impressions Scale (CGI-I and CGI-S, respectively).

- The clinicians' impression of illness severity and symptom improvement and patient- or caregiver-reported impressions of general functioning and sleep also were noted.

\section{Outcomes}

- After 6 weeks, compared with the placebo group, the CBD group had lower levels of positive psychotic symptoms and were more likely to be rated as improved and as not severely unwell by the treating clinician. Patients in the CBD group also showed greater improvements in cognitive performance and in overall functioning, although these were not statistically significant.

- Similar levels of negative psychotic symptoms, overall psychopathology, and general psychopathology were observed in the CBD and placebo groups. The CBD group had a higher proportion of treatment responders $(\geq 20 \%$ improvement in PANSS

\section{Clinical Point}

CBD does not appear to involve the direct antagonism of dopamine receptors; however, its mechanism of action is unclear 


\section{Clinical Point}

After 6 weeks of $C B D$, $1,000 \mathrm{mg} / \mathrm{d}$, patients had lower levels of positive psychotic symptoms than those who received placebo

\section{Table}

\section{CBD for psychosis: 4 Studies}

\begin{tabular}{|c|c|c|}
\hline Study & Design & Outcomes \\
\hline McGuire et al ${ }^{4}$ & $\begin{array}{l}\text { Six-week trial of } 88 \text { patients with } \\
\text { schizophrenia randomized to receive } \\
\text { CBD, } 1,000 \mathrm{mg} / \mathrm{d} \text {, or placebo in } \\
\text { addition to their usual antipsychotic }\end{array}$ & $\begin{array}{l}\text { Compared with the placebo group, the } \\
\text { CBD group had lower levels of positive } \\
\text { psychotic symptoms and were more likely } \\
\text { to be rated as improved by the treating } \\
\text { clinician }\end{array}$ \\
\hline Boggs et al ${ }^{5}$ & $\begin{array}{l}\text { Six-week trial of } 36 \text { stable patients } \\
\text { with schizophrenia treated with } \\
\text { antipsychotics who were randomized } \\
\text { to receive CBD, } 600 \mathrm{mg} / \mathrm{d} \text {, or placebo }\end{array}$ & $\begin{array}{l}\text { Compared with placebo, CBD } \\
\text { augmentation did not improve cognition } \\
\text { or positive psychotic symptoms }\end{array}$ \\
\hline O'Neill et al ${ }^{6}$ & $\begin{array}{l}\text { Using fMRI, researchers evaluated } \\
\text { brain activation during a verbal } \\
\text { learning task in } 15 \text { patients with } \\
\text { psychosis treated with antipsychotics } \\
3 \text { hours after they received a single } \\
600 \text {-mg dose of CBD, and in } 19 \\
\text { healthy controls who did not receive } \\
\text { any CBD or medication }\end{array}$ & $\begin{array}{l}\text { Altered activation in the prefrontal and } \\
\text { mediotemporal regions during verbal } \\
\text { learning in patients with psychosis } \\
\text { appeared to be partially normalized after } \\
\text { a single } 600 \text {-mg dose of CBD }\end{array}$ \\
\hline $\begin{array}{l}\text { Bhattacharyya } \\
\text { et } \mathrm{al}^{7}\end{array}$ & $\begin{array}{l}\text { Researchers used fMRI to evaluate } \\
\text { brain activation during a verbal } \\
\text { learning task in } 33 \text { antipsychotic- } \\
\text { naïve patients at clinical high risk for } \\
\text { psychosis who were randomized to } \\
\text { receive a single } 600 \text {-mg dose of CBD } \\
\text { or placebo, and in } 19 \text { healthy controls } \\
\text { who did not receive any CBD or } \\
\text { medication }\end{array}$ & $\begin{array}{l}\text { Relative to control participants, high-risk } \\
\text { participants exhibited different levels } \\
\text { of activation in several brain regions, } \\
\text { including } 3 \text { believed to be critical to the } \\
\text { pathophysiology of psychosis (striatum } \\
\text { during verbal encoding, and medial } \\
\text { temporal lobe and midbrain during verbal } \\
\text { recall). At-risk participants who received } \\
\text { CBD had greater levels of brain activation } \\
\text { and higher recall }\end{array}$ \\
\hline
\end{tabular}

total score) than did the placebo group; however, the total number of responders per group was small (12 and 6 patients, respectively). At baseline, most patients in both groups were classified as moderately, markedly, or severely ill (83.4\% in the CBD group vs $79.6 \%$ in placebo group). By the end of treatment, this decreased to $54.8 \%$ in the CBD group and $63.6 \%$ in the placebo group. Clinicians rated $78.6 \%$ of patients in the CBD group as "improved" on the CGI-I, compared with $54.6 \%$ of patients in the placebo group.

\section{Conclusion}

- CBD treatment adjunctive to antipsychotics was associated with significant effects on positive psychotic symptoms and on CGI-I and illness severity. Improvements in cognitive performance and level of overall functioning were also seen, but were not statistically significant.

- Although the effect on positive symptoms was modest, improvement occurred in patients being treated with appropriate dosages of antipsychotics, which suggests CBD provided benefits over and above the effect of antipsychotic treatment. Moreover, the changes in CGI-I and CGI-S scores indicated that the improvement was evident to the treating psychiatrists, and may therefore be clinically meaningful.

2. Boggs DL, Surti T, Gupta A, et al. The effects of cannabidiol (CBD) on cognition and symptoms in outpatients with chronic schizophrenia a randomized placebo controlled trial. Psychopharmacology (Berl). 2018;235(7):1923-1932. 
Schizophrenia is associated with cognitive deficits in learning, recall, attention, working memory, and executive function. The cognitive impairments associated with schizophrenia (CIAS) are independent of phase of illness and often persist after other symptoms have been effectively treated. These impairments are the strongest predictor of functional outcome, even more so than psychotic symptoms.

Antipsychotics have limited efficacy for CIAS, which highlights the need for CIAS treatments that target other nondopaminergic neurotransmitter systems. The endocannabinoid system, which has been implicated in schizophrenia and in cognition, is a potential target. Several cannabinoids impair memory and attention. The main psychoactive component of marijuana, THC, is a cannabinoid receptor type 1 (CB1R) partial agonist. Administration of THC produces significant deficits in verbal learning, attention, and working memory.

Researchers have hypothesized that CB1R blockade or modulation of cannabinoid levels may offer a novel target for treating CIAS. Boggs et $\mathrm{al}^{5}$ compared the cognitive, symptomatic, and adverse effects of $C B D$ vs placebo.

\section{Study design}

- In this 6-week, randomized, placebocontrolled study conducted in Connecticut from September 2009 to May 2012, 36 stable patients with schizophrenia who were treated with antipsychotics were randomized to also receive oral CBD, $600 \mathrm{mg} / \mathrm{d}$, or placebo.

- Cognition was assessed using the $t$ score of the MATRICS Consensus Cognitive Battery (MCCB) composite and subscales at baseline and the end of study. An increase in MCCB $t$ score indicates an improvement in cognitive ability. Psychotic symptoms were assessed using the PANSS at baseline, Week 2, Week 4, and Week 6.

\section{Outcomes}

- CBD augmentation did not improve MCCB performance or psychotic symptoms.
There was no main effect of time or medication on MCCB composite score, but a significant drug $\times$ time effect was observed.

- Post-hoc analyses revealed that only patients who received placebo improved over time. The lack of a similar improvement with CBD might be related to the greater incidence of sedation among the CBD group (20\%) vs the placebo group (5\%). Both the MCCB composite score and reasoning and problem-solving domain scores were higher at baseline and endpoint for patients who received $C B D$, which suggests that the observed improvement in the placebo group could represent a regression to the mean.

- There was a significant decrease in PANSS scores over time, but there was no significant drug $\times$ time interaction.

\section{Conclusion}

- CBD augmentation was not associated with an improvement in MCCB score. This is consistent with data from other clinical trials $^{4,8}$ that suggested that CBD (at a wide range of doses) does not have significant beneficial effects on cognition in patients with schizophrenia.

- Additionally, CBD did not improve psychotic symptoms. These results are in contrast to published case reports ${ }^{9,10}$ and 2 published clinical trials ${ }^{3,4}$ that found CBD $(800 \mathrm{mg} / \mathrm{d})$ was as efficacious as amisulpride in reducing positive psychotic symptoms, and a small but statistically significant improvement in PANSS positive scores with CBD $(1,000$ $\mathrm{mg} / \mathrm{d}$ ) compared with placebo. However, these results are similar to those of a separate study $^{11}$ that evaluated the same $600-\mathrm{mg} / \mathrm{d}$ dose of CBD used by Boggs et al. ${ }^{5}$ At 600 $\mathrm{mg} / \mathrm{d}, \mathrm{CBD}$ produced very small improvements in PANSS total scores ( 2.4) that were not statistically significant. A higher CBD dose may be needed to reduce psychotic symptoms in patients with schizophrenia.

3. O'Neill A, Wilson R, Blest-Hopley G, et al. Normalization of mediotemporal and prefrontal activity, and mediotemporal-striatal connectivity, may underlie antipsychotic effects of

\section{Clinical Point}

In a 6-week study, CBD, $600 \mathrm{mg} / \mathrm{d}$, did not improve cognition or psychotic symptoms in 36 stable patients with schizophrenia 


\section{Clinical Point}

Altered activation
in prefrontal and
mediotemporal
regions in 15 patients
with psychosis was
partially normalized
after 1 dose of CBD

cannabidiol in psychosis. Psychol Med. 2020; 1-11. doi: 10.1017/S0033291719003519.

In addition to their key roles in the psychopathology of psychosis, the mediotemporal and prefrontal cortices are involved in learning and memory, and the striatum plays a role in encoding contextual information associated with memories. Because deficits in verbal learning and memory are one of the most commonly reported impairments in patients with psychosis, $\mathrm{O}^{\prime} \mathrm{Neill}$ et $\mathrm{al}^{6}$ used functional MRI (fMRI) to examine brain activity during a verbal learning task in patients with psychosis after taking CBD or placebo.

\section{Study design}

- In a double-blind, randomized, placebocontrolled, crossover study, researchers investigated the effects of a single dose of CBD in 15 patients with psychosis who were treated with antipsychotics. Three hours after taking a 600-mg dose of CBD or placebo, these participants were scanned using fMRI while performing a verbal paired associate (VPA) learning task. Nineteen healthy controls underwent $\mathrm{AMRI}$ in identical conditions, but without any medication administration.

- The fMRI measured brain activation using the blood oxygen level-dependent (BOLD) hemodynamic responses of the brain. The fMRI signals were studied in the mediotemporal, prefrontal, and striatal regions.

- The VPA task presented word pairs visually, and the accuracy of responses were recorded online. The VPA task was comprised of 3 conditions: encoding, recall, and baseline.

- Results during each phase of the VPA task were compared.

\section{Outcomes}

- While completing the VPA task after taking placebo, compared with healthy controls, patients with psychosis demonstrated a different pattern of activity in the prefrontal and mediotemporal brain areas. Specifically, during verbal encoding, the placebo group showed altered activation in prefrontal regions. During verbal recall, the placebo group showed altered activation in prefrontal and mediotemporal regions, as well as increased mediotemporal-striatal functional connectivity.

- After participants received CBD, activation in these brain areas became more like the activation seen in controls. CBD attenuated dysfunction in these regions such that activation was intermediate between the placebo condition and the control group. CBD also attenuated functional connectivity between the hippocampus and striatum, and lead to reduced symptoms in patients with psychosis (as measured by PANSS total score).

\section{Conclusion}

- Altered activation in prefrontal and mediotemporal regions during verbal learning in patients with psychosis appeared to be partially normalized after a single $600-\mathrm{mg}$ dose of CBD. Results also showed improvement in PANSS total score with CBD.

- These findings suggest that a single dose of CBD may partially attenuate the dysfunctional prefrontal and mediotemporal activation that is believed to underlie the dopamine dysfunction that leads to psychotic symptoms. These effects, along with a reduction in psychotic symptoms, suggest that normalization of altered prefrontal and mediotemporal function and mediotemporal-striatal connectivity may underlie the antipsychotic effects of CBD in established psychosis.

\section{Bhattacharyya S, Wilson R, Appiah-Kusi E, et al. Effect of cannabidiol on medial tem- poral, midbrain, and striatal dysfunction in people at clinical high risk of psychosis: a randomized clinical trial. JAMA Psychiatry. 2018;75(11):1107-1117.}

Current preclinical models suggest that psychosis involves a disturbance of activity in the medial temporal lobe (MTL) that drives dopamine dysfunction in the striatum and midbrain. THC, which produces psychotomimetic effects, impacts the function of the 
striatum (verbal memory and salience processing) and amygdala (emotional processing), and alters the functional connectivity of these regions. Compared with THC, CBD has broadly opposite neural and behavioral effects, including opposing effects on the activation of these regions. Bhattacharyya et $\mathrm{al}^{7}$ examined the neurocognitive mechanisms that underlie the therapeutic effects of CBD in psychosis and sought to understand whether CBD would attenuate functional abnormalities in the MTL, midbrain, and striatum.

\section{Study design}

- A randomized, double-blind, placebocontrolled trial examined 33 antipsychoticnaïve participants at clinical high risk (CHR) for psychosis and 19 healthy controls. The CHR group was randomized to CBD, $600 \mathrm{mg}$, or placebo.

- Three hours after taking CBD or placebo, CHR participants were studied using fMRI while performing a VPA learning task, which engages verbal learning and recall in the MTL, midbrain and striatum. Control participants did not receive any medication but underwent fMRI while performing the VPA task.

- The VPA task presented word pairs visually, and the accuracy of responses was recorded online. It was comprised of 3 conditions: encoding, recall, and baseline.

\section{Outcomes}

- Brain activation was analyzed in 15 participants in the CBD group, 16 in the placebo group, and 19 in the control group. Activation during encoding was observed in the striatum (specifically, the right caudate). Activation during recall was observed in the midbrain and the MTL (specifically, the parahippocampus).

- Brain activation levels in all 3 regions were lowest in the placebo group, intermediate in the CBD group, and greatest in the healthy control group. For all participants, the total recall score was directly correlated with the activation level in the left MTL (parahippocampus) during recall.

\section{Conclusion}

- Relative to controls, CHR participants exhibited different levels of activation in several regions, including the 3 areas thought to be critical to the pathophysiology of psychosis: the striatum during verbal encoding, and the MTL and midbrain during verbal recall.

- Compared with those who received placebo, CHR participants who received CBD before completing the VPA task demonstrated greater levels of brain activation and higher recall score.

- These findings suggest that CBD may partially normalize alterations in MTL, striatal, and midbrain function associated with $\mathrm{CHR}$ of psychosis. Because these regions are implicated in the pathophysiology of psychosis, the impact of CBD at these sites may contribute to the therapeutic effects of CBD that have been reported by some patients with psychosis.

\section{Conflicting data highlights the need for longer, larger studies}

Research findings on the use of CBD for psychotic symptoms in patients with schizophrenia have been conflicting. Some early research suggests that taking CBD 4 times daily for 4 weeks improves psychotic symptoms and might be as effective as the antipsychotic amisulpride. However, other early research suggests that taking CBD for 14 days is not beneficial. The conflicting results might be related to the CBD dose used and duration of treatment.

Davies and Bhattacharya ${ }^{12}$ recently reviewed evidence regarding the efficacy of CBD as a potential novel treatment for psychotic disorders. They concluded that CBD represents a promising potential novel treatment for patients with psychosis. It also appears that CBD may improve the disease trajectory of individuals with early psychosis and comorbid cannabis misuse. ${ }^{13} \mathrm{CBD}$ use has also been associated with a decrease in symptoms of psychosis and changes in brain activity during verbal memory tasks in patients at high risk of psychosis. ${ }^{6}$

\section{Clinical Point}

In patients at high risk for psychosis, CBD may partially normalize brain function alterations associated with psychosis 


\section{Clinical Point}

\section{CBD may improve} the disease trajectory in patients with early psychosis and comorbid cannabis misuse
However, before CBD can become a viable treatment option for psychosis, the promising findings in these initial clinical studies must be replicated in large-scale trials with appropriate treatment duration.

\section{References}

1. US National Library of Medicine. MedlinePlus. Cannabidiol (CBD). https://medlineplus.gov/druginfo/natural/1439. html. Accessed May 14, 2020.

2. Bonn-Miller MO, Loflin MJE, Thomas BF, et al. Labeling accuracy of cannabidiol extracts sold online. JAMA. 2017;318(17):1708-1709.

3. Leweke FM, Piomelli D, Pahlisch F, et al. Cannabidiol enhances anandamide signaling and alleviates psychotic symptoms of schizophrenia. Transl Psychiatry. 2012;2(3):e94.

4. McGuire P, Robson P, Cubala WJ, et al. Cannabidiol (CBD) as an adjunctive therapy in schizophrenia: a multicenter randomized controlled trial. Am J Psychiatry. 2018;175(3):225-231.

5. Boggs DL, Surti T, Gupta A, et al. The effects of cannabidiol (CBD) on cognition and symptoms in outpatients with chronic schizophrenia a randomized placebo controlled trial. Psychopharmacology (Berl). 2018;235(7):1923-1932.

6. O'Neill A, Wilson R, Blest-Hopley G, et al. Normalization of mediotemporal and prefrontal activity, and mediotemporalstriatal connectivity, may underlie antipsychotic effects of cannabidiol in psychosis. Psychol Med. 2020;1-11. doi: 10.1017/S0033291719003519.

7. Bhattacharyya S, Wilson R, Appiah-Kusi E, et al. Effect of cannabidiol on medial temporal, midbrain, and striatal dysfunction in people at clinical high risk of psychosis: a randomized clinical trial. JAMA Psychiatry. 2018;75(11): 1107-1117.

8. Hallak JE, Machado-de-Sousa JP, Crippa JAS, et al. Performance of schizophrenic patients in the Stroop color word test and electrodermal responsiveness after acute administration of cannabidiol (CBD). Rev Bras Psiquiatr. 2010;32(1):56-61.

9. Zuardi AW, Morais SL, Guimaraes FS, et al. Antipsychotic effect of cannabidiol. J Clin Psychiatry. 1995;56(10): 485-486.

10. Zuardi AW, Hallak JE, Dursun SM, et al. Cannabidiol monotherapy for treatment-resistant schizophrenia. J Psychopharmacol. 2006;20(5):683-686.

11. Leweke FM, Hellmich M, Pahlisch F, et al. Modulation of the endocannabinoid system as a potential new target in the treatment of schizophrenia. Schizophr Res. 2014; 153(1):S47.

12. Davies C, Bhattacharyya S. Cannabidiol as a potential treatment for psychosis. Ther Adv Psychopharmacol. 2019, 9. doi: $10.1177 / 2045125319881916$.

13. Hahn B. The potential of cannabidiol treatment for cannabis users with recent-onset psychosis. Schizophr Bull. 2018;44(1):46-53 